\title{
ANÁLISIS DEL RELACIONAMIENTO DE LOS GRUPOS HUMANOS CON EL BOSQUE DESDE LOS PRINCIPIOS DE OSTROM
}

\author{
THE RELATIONSHIP BETWEEN SOCIAL GROUPS \\ AND THE FOREST: AN ANALYSIS USING OSTROM'S PRINCIPLES
}

\author{
1 María Fernanda Franco Ortiz \\ 2 Jaime Alberto Moreno Gutiérrez \\ ${ }^{1}$ Magister en Manejo, Uso y Conservación del Bosque, Universidad Distrital FJdeC, Bogotá- Colombia \\ ${ }^{2}$ Doctorante Pensamiento Complejo - Multiversidad Mundo Real; Profesor Asociado \\ Universidad Distrital FJdC, Bogotá- Colombia \\ ${ }^{1}$ mariaffrancoo@gmail.com \\ 2 jmoreno@udistrital.edu.co
}

\section{RESUMEN}

Desde una perspectiva gubernamental, donde los Estados son los propietarios de los bosques, los marcos regulatorios para el manejo forestal son típicamente asimétricos en relación con la dinámica de la comunidad local. Esta investigación pretende identificar, a partir de estudios de caso, la forma de relacionamiento del ser humano con el bosque para establecer los aspectos favorables y las barreras que enfrenta la materialización de la autogestión como la base de una gobernanza efectiva en el manejo de los bosques naturales en los trópicos. Se abordó la revisión de 60 estudios de caso, de los cuales tan solo 26 presentan una sistematización conceptual con rasgos específicos de los principios planteados por Ostrom para los RUC. Con este análisis, se encontró que le principio más recurrente en los estudios es la "coherencia entre reglas de apropiación y provisión" y el menos considerado es el "monitoreo de recursos", lo cual deja en evidencia que las comunidades siempre establecen reglas, pero no tienen clara la importancia del monitoreo.

Palabras clave: Autogestión de los bosques; Complejidad Forestal; Gestión compleja; Gestión de bosques; Gobernanza forestal; Recursos de Uso Común (RUC)

\section{ABSTRACT \\ From a governmental perspective, where the state is the owner of forests, regulatory fra- meworks for forest management are typically}


asymmetric in relation to the dynamics of the local community. This research identifies, by using study cases, the relationship form of the human being with the forest, in order to establish the favorable aspects and the barriers that face the materialization of the self-management as the base of effective governance in the management of the natural tropical forests. The review of 60 case studies was addressed, of which only 26 present a conceptual systematization with specific features of the principles proposed by Ostrom for "resources of common use" (RCU). The analysis concludes that the most recurrent principle in the studies is the "coherence between rules of appropriation and provision", while the least considered is the "monitoring of resources", which shows that communities always establish rules; however, the importance of monitoring is not clear for them.

Keywords: Forest Management; Commonpool Resource (CPR); Self-management of Forests; Forest Governance; Forestry Complexity; Complex Management

\section{INTRODUCCIÓN}

Los recursos de uso común (RUC) se caracterizan por ser recursos sobre los cuales es muy difícil o costoso excluir a alguien de su uso o beneficio; sin embargo, al mismo tiempo presentan rivalidad, dado que la porción del recurso que usa una persona ya no estará disponible posteriormente para ser usada por otra, al menos en el corto y mediano plazo, ocasionando que el recurso pueda llegar a sobreexplotarse (Bollier, 2014; Cardenas, 2009; Ostrom, 2011). En el caso específico de los bosques naturales, estos son considerados como RUC puesto que ya sea de facto o iure se accede y usan, inmersos al mismo tiempo en una variedad de derechos no necesariamente equilibrados en cuanto a deberes o precauciones sobre la forma y medio de dicho uso (Cardenas, 2009; E. Ostrom, 2009). Diferentes autores como Meinzen-Dick \& Di Gregorio (2004), Ostrom (2009a), Schlager \& Ostrom (1992), han trabajado sobre la clasificación y conceptualización de unos "derechos de propiedad" facticos, que corresponden principalmente a cinco aspectos para su configuración: acceso, retiro (extracción), gestión, exclusión y alienación.
Garrett Hardin (1915- 2003) fue uno de los primeros autores que consideró la necesidad de contar con derechos de propiedad privada para garantizar la sostenibilidad de un RUC, ya sea bajo una regulación centralizada a través de empresas privadas o bajo el control del gobierno (Hardin 1968; Delgado Riaño, 2017; Gómez \& Guerrero, 2014). Su exposición en "la tragedia de los comunes" consistía en argumentar que los grupos humanos no eran capaces de trabajar juntos de manera efectiva, puesto que el principio de maximización individual del beneficio incentivaba a la sobreexplotación a costa de la restricción de usufructo de otros, frente o futuro, del mismo recurso (Olson 1965; Hardin 1968).

El postulado de Hardin resultó alentador para el desarrollo de investigaciones. Una de las más representativas fue la llevada a cabo por Elinor Ostrom, pues desarrolló un marco teórico a partir de un exhaustivo análisis de "realidades empíricas" en el que, por más de 30 años, documentó y analizó con casos alrededor del mundo con respecto a la gestión de RUC. Su investigación la llevó a ganar el premio nobel 
de economía en el 2009, comprendiendo y demostrando cómo algunas comunidades son capaces de gestionar sus recursos de manera exitosa, sin contar necesariamente con un derecho de propiedad explicito, mientras que otras no (aun en presencia de dicho derecho), sin importar si los recursos son gestionados bajo esquemas de tenencia privada o por el Estado, sin ser ello la clave que conduzca a una gestión sostenible (Ostrom, 2011).

Es importante tener en cuenta que, más allá de los derechos de propiedad que se tienen sobre las áreas donde están los recursos, localmente se dan dinámicas sobre la explotación o aprovechamiento del bosque, donde los actores cuentan con diferentes niveles de elección colectiva que influyen directamente en los regímenes de uso y gestión de los mismos (Schlager \& Ostrom, 1992; Cardenas, 2009; Ostrom, 2011). Frente a estas realidades, resulta relevante conocer, identificar y construir, a partir de este reconocimiento, las directrices de gestión efectiva de los bosques, principalmente cuando se reconoce que los marcos normativos gubernativos difieren en gran medida de las realidades locales, que los hacen ineficientes; por lo tanto, en esta investigación se identifican los elementos facticos de la relación de los individuos con el bosque.

\section{PRINCIPIOS DE DISEÑO CARACTERÍSTI- COS DE INSTITUCIONES DE RUC}

La investigación en campo sobre los RUC, ha permitido un gran avance en la conceptualización y teorización de estos(Poteete, Janssen, \& Ostrom, 2012). El trabajo de Elinor Ostrom ha sido la base para el desarrollo de este tema; a partir de su libro "El Gobierno de los Bienes Comunes" (Ostrom, 2011), en el cual recorrió y estudió una variedad de sistemas de recursos de uso común. Ella definió ocho principios clave presentes en estos sistemas, los cuales expone como algunas condiciones que contribuyen al éxito de la gestión efectiva de los RUC.

A continuación, se presentan los Principios definidos por Ostrom para el desarrollo de investigaciones relacionadas con los RUC.

\section{Límites claramente definidos}

Los límites de un sistema de recursos deben ser claros (cuánto se puede usar) y se deben conocer los usuarios que están autorizados para usar el recurso (quienes lo usan) (Elinor Ostrom, 2009a). Los niveles de confianza y de cooperación aumentan cuando se tiene menos incertidumbre y mayor conocimiento del recurso por usar, esta información permite construir reglas sobre los límites del recurso, la autorización de acceso, la cosecha, la administración y la exclusión (Elinor Ostrom, 2009; Ostrom, 2011). Algunos investigadores sugieren que, para logar una mayor efectividad en la aplicación de este principio, este se debe dividir en dos partes: una centrada en los límites de los actores autorizados para utilizar un recurso de propiedad común y el otro relacionado con los límites del propio recurso (Agrawal 2002; Cox, Arnold, \& Villamayor, 2010). Los límites para los usuarios deben dejar claro quiénes son los usuarios legítimos. Los límites de los recursos definen el sistema de recursos y ayuda a precisar su entorno biofísico.

\section{Coherencia entre las reglas de apropiación y provisión con las condiciones locales}

Las reglas de uso se relacionan con las unidades del recurso por adquirir y las variables afines a esa actividad, esas a su vez deben estar creadas por los usuarios de acuerdo con la realidad, contexto y entorno de los mismos. Estas reglas son específicas para cada recurso y sus usuarios (Ostrom, 2009; Ostrom, 2011). Generalmente, las reglas que se conciben desde un nivel local, tienen menor incertidumbre sobre los recursos y las dinámicas en torno a 
este. Por lo anterior, puede ser más sencillo entender las reglas, y su aplicación por parte de los usuarios. También será mayor la probabilidad de las autoridades locales de propiciar un gobierno eficaz de los recursos (Agrawal, 2007; C. C. Gibson, Williams, \& Ostrom, 2005). En este principio, autores como (Cox et al., 2010) sugieren una división del mismo y así lograr una mayor profundidad en la gestión. Las reglas de apropiación y provisión hacen referencia a la distribución de beneficios obtenidos del aprovechamiento del recurso conforme a la distribución de los costos de provisión, mientras que la congruencia con las condiciones locales busca que las reglas sean adecuadas con las condiciones socio-ambientales locales.

\section{Arreglos de elección colectiva}

Los diferentes grupos de personas que utilizan un recurso son aptos para crear sus propios arreglos, dado que conocen e interactúan permanentemente en el mismo entorno. Además, tienen la posibilidad de ir adaptando y reformulando las normas con el tiempo, pues la dinámica alrededor del recuso no siempre será la misma (Elinor Ostrom, 2009; Ostrom, 2011). Cuando los usuarios son los que toman las decisiones sobre las normas, es más probable que estas se cumplan y se hagan cumplir, debido a que su planteamiento es desde una visión cercana al recurso y sus dinámicas, en comparación con situaciones donde las normas son impuestas por personas ajenas a la comunidad. El reto en ese momento para los grupos de usuarios, es que es más fácil establecer normas que cumplirlas, dado que se pueden presentar diversas tentaciones que afecten los intereses (Elinor Ostrom \& Nagendra, 2006; Ostrom, 2011).

\section{Monitoreo}

El monitoreo sobre los RUC hace referencia a los métodos aplicados para contener la trasgresión de las normas establecidas por los usuarios. En muchos de los regímenes de RUC, las personas que monitorean la mayoría de veces son de la misma comunidad (Elinor Ostrom, 2009a; Ostrom, 2011), lo que resulta sostenible económicamente ya que los apropiadores reducen los costos de esta actividad (Olivos, 2014). Cox et al (2010), consideran que, con el objetivo de tener una mayor efectividad con este principio, se debe dividir en dos subtemas: i. El monitoreo de los usuarios en donde los monitores vigilan los niveles de apropiación y provisión del recurso por parte de las personas que acceden al uso del recurso; ii. El monitoreo sobre el recurso, donde se controlan las condiciones del mismo.

\section{Sanciones graduadas}

Las sanciones graduadas hacen referencia a la necesidad de crear un sistema de sanciones que estén de acuerdo con la gravedad y el contexto de la infracción, así como su repetición. De esta forma, las personas que incumplen una norma serán sancionadas según la gravedad de la falta cometida, pero así mimo queda en conocimiento de todos la sanción que se daría si este usuario cometiera de nuevo la misma infracción (una sanción más fuerte). Esto aumenta la confianza de los usuarios con respecto a los incumplimientos cometidos por otras personas (Elinor Ostrom, 2009a; Ostrom, 2011). Este principio se relaciona íntimamente con el de monitoreo pues existe una relación en ambas direcciones. Como se mencionaba, se busca que los mismos usuarios sean los que monitorean ya que, además de los beneficios de sostenibilidad económica, el conocimiento que los usuarios tienen del recurso, las dinámicas, las reglas y la información de cumplimiento de los demás participantes, permite un mejor seguimiento (Ostrom, 2011). Será más difícil que un infractor reincida en su falta si esta ya está en conocimiento de toda la comunidad y las personas que monitorean; además, la reputación frente a la comunidad cobra un 
papel importante en las motivaciones comportamentales de los usuarios.

\section{Mecanismos para la resolución de conflictos}

Los conflictos frente a la gestión de RUC están relacionados en su mayoría por la interpretación de las normas, la búsqueda es el funcionamiento armónico del sistema de recursos para que este permanezca en tiempo y espacio. Lo anterior incide de igual manera en la necesidad de tener un buen diseño de las normas que rigen el sistema, pues de esta depende su interpretación y cumplimiento en la práctica, de no ser así, surgen los conflictos por la interpretación (Olivos, 2014). El diseño de un mecanismo simple, local y de fácil acceso para todas las personas, permitirá solucionar los conflictos rápidamente. Este mecanismo facilita las acciones de la comunidad y le genera una mayor confianza interna, ya que se utiliza para mediar conflictos entre usuarios o con funcionarios y es de pleno conocimiento de todos los usuarios (Elinor Ostrom, 2009a; Ostrom, 2011).

\section{Reconocimiento mínimo de derechos de organización}

"Los derechos de los apropiadores para elaborar sus propias instituciones no son cuestionados por autoridades gubernamentales externas" (Ostrom, 2011).

Los regímenes de organización y gestión sobre el recurso creados por los propios usuarios generan una mayor eficacia dado el conocimiento de las dinámicas. La intervención e imposición por actores externos, puede generar conflictos sobre el recurso y en las dinámicas sociales, dado el desconocimiento y las formas de intervención externas (Elinor Ostrom, 2009a; Elinor Ostrom \& Nagendra, 2006). Los usuarios de los recursos deben contar con la autonomía para crear sus normas; sin embargo, así mismo se espera el respaldo de autoridades externas que reconozcan y les permitan hacer valer sus derechos. Las autoridades externas deben conocer las reglas de las organizaciones de usuarios ya que esto fortalece las organización y a las reglas propiamente dichas (Olivos, 2014).

\section{Entidades anidadas}

Las entidades anidadas hacen referencia a una serie de organizaciones que están incluidas unas en otras. Es decir, la toma de decisiones y el control de los sistemas de recursos resultan complejos si se san desde un mismo nivel, razón por la cual se crean organizaciones encargadas de la gestión en diferentes niveles. Los principios expuestos anteriormente deben encontrarse en instituciones cada vez mayores que vayan acogiendo las de niveles inferiores y así nutrir los sistemas y generar unos mejores ejercicios de gobernanza (C. M. Tucker, 2010) acordes con las realidades desde lo más local a mayores unidades de gestión (Elinor Ostrom, 2009a; Ostrom, 2011).

\section{METODOLOGÍA}

La presente investigación se basa en la Teoría Fundamentada (Glaser y Strauss, 1967), donde, a partir de un muestreo teórico, se fundamenta conceptualmente la base de la gestión de los RUC, para posteriormente, y con base en el rastreo de casos publicados, reconocidos y aceptados como consistentes, hacer la constatación o verificación de evidencia explícita o implícita de algunos o todos los factores de éxito para la gestión efectiva de los RUC, esgrimidos por Ostro.

Reconociendo los fundamentos y desarrollos conceptuales alrededor de la gestión de los bosques, se buscaba identificar unos parámetros que aborden los diferentes elementos que componen y describen las relaciones (en sus múltiples niveles) que tiene las personas y las instituciones con la gestión de los bosques. 
Para esta selección, fue importante tener en cuenta que existen múltiples formas de relacionamiento con los bosques por parte de las personas, donde justamente se plantean los conflictos en las decisiones normativas que regulan el uso de los recursos por parte de los gobiernos; por lo anterior, tras la revisión bibliográfica se consideró que los parámetros planteados por Ostrom (1990) resultan suficientes para enmarcar la información que se busca en esta investigación. Se seleccionaron estos parámetros considerando las modificaciones propuestas por Cox et al (2010) debido a que son parámetros construidos desde una base real del análisis de casos de gestión de los recursos, en donde su autora recogió experiencias alrededor del mundo por más de 30 años, llegando a abstraer estos parámetros desde las particularidades propias de los sistemas de gestión de los recursos.

Basados en los principios, se desarrolló una búsqueda de casos en revistas indexadas, así como en los repositorios de universidades. La búsqueda de estos casos se basó en descriptores claves como: RUC, gestión de los bosques, manejo de bosques y principios de gestión de bosques. La búsqueda de casos permitió identificar más de 60 intentos de sistematización, los cuales posteriormente se revisaron, buscando que hicieran una descripción de los modelos locales de gestión del bosque, que saltaran de la simple relatoría a un análisis conceptual que permitiera vislumbrar, explícita o implícitamente, al menos 4 de los 10 principios establecidos para la investigación; así mismo, la muestra se tomó con base en que fueran estudios de caso reportados en los trópicos, debido a la afinidad de contextos tanto naturales como sociales que se pueden presentar con el contexto colombiano. Los casos que cumplían con estos criterios pasaban a una revisión posterior, mientras que los demás eran descartados.
A partir de la información brindada por cada caso, se desarrolló una matriz en donde cada columna hacía referencia a cada uno de los principios y a la información general del estado de caso, en las filas se iban registrando cada uno de los casos que cumplían con los parámetros. Por medio de este trabajo se obtuvo una matriz de llenos y vacíos en donde se identifican los principios desarrollados en cada estudio de caso.

Con la selección de fragmentos de textos en los cuales se desarrollan los principios en cada uno de los casos, se procedió a determinar "códigos" a partir de la información allí contenida. Los códigos son unas etiquetas que permiten sintetizar la información allí contenida y da insumos para conocer los diferentes panoramas que se pueden dar sobre un principio. Estos códigos se trabajaron con el objetivo de establecer categorías en cada uno de los principios que en los que se integraron los diferentes modos de relacionamiento entre el ser humano y la naturaleza.

\section{RESULTADOS Y DISCUSIÓN}

Con la revisión de los estudios se pudo establecer universo muestral de 60 casos de sistematización de experiencias relacionados con la sociedad o grupos de colectivos y el recurso forestal, desarrollando de manera explícita o implícita mecanismos de gestión, derivados de su relacionamiento vivencial con el bosque. Se identificaron 26 estudios de caso distribuidos por los trópicos, en los que las sistematizaciones presentaban rasgos específicos de los planteamientos que Ostrom (2011) desarrolla sobre la gestión de los RUC, que incluyen con claridad al menos cuatro de ellos definidos previamente como aparataje conceptual de esta investigación.

Dentro de los casos seleccionados, se permitió contar con varios actores que describieran sus 
formas de gestión y relacionamiento con los bosques. La forma como estos grupos humanos conciben el bosque difiere, puesto que los valores que le dan se relacionan directamente con la historia de uso y la forma de relacionamiento que han tenido. Por tal razón, para tener un marco más amplio sobre el relacionamiento de grupos de usuarios con el bosque, se consideró que la inclusión de casos que cubriera diferentes actores.

\section{Identificación de parámetros}

Con la sistematización de todos los casos se realizó un análisis de la respuesta de la muestra frente a los principios establecidos (Figura 1). El principio que se encuentra menos establecido es el monitoreo de los recursos, ya que desde los casos revisados, el hecho de monitorear los recursos incurre en costos muy altos que muchas veces las comunidades no pueden asumir. De la misma forma, se observa que no hay claridad sobre la aplicación de este principio, pues no se refiere únicamente a tener vigilancia del bosque sino monitorear la respuesta del bosque (tanto en su estructura, composición y función) a las actividades que allí se realizan.

El principio con el valor más alto es "coherencia entre reglas apropiación y provisión", debido a que, ya sea por acuerdos implícitos o explícitos y sin importar las particularidades culturales y de manejo de los recursos, la gran mayoría de grupos de usuarios establecen normas para la gestión del recurso boscoso. El hecho que las comunidades tengan un relacionamiento directo, y muchas veces histórico con el recurso, disminuye en ellas la incertidumbre sobre el recurso en sí, así como su dinámica, lo que les permite tomar decisiones fundamentadas sobre las reglas para la gestión. Cabe resaltar que en algunos casos esas reglas vienen impuestas desde niveles superiores de gestión, lo que llega a condicionar, en ciertos casos el cumplimiento de las mismas por parte de los usuarios.

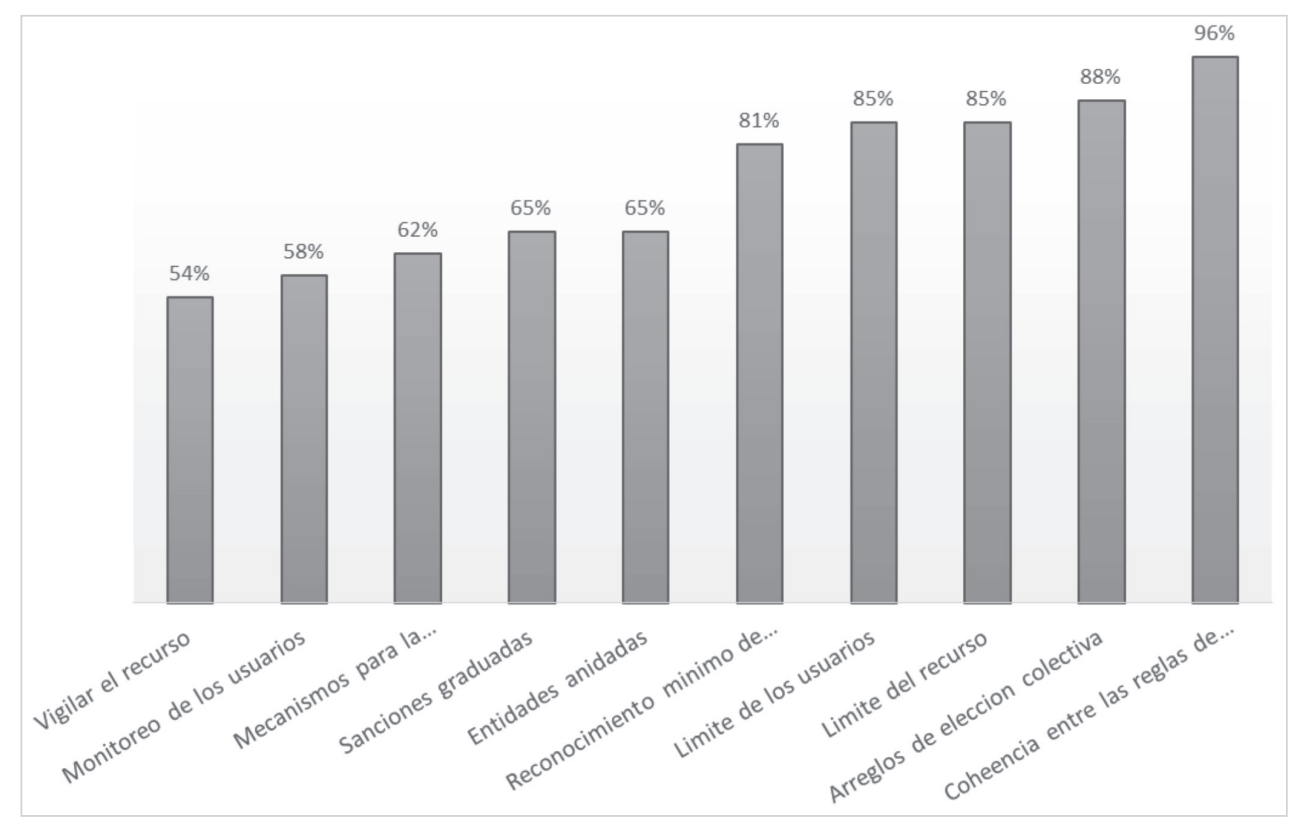

Figura 1. Porcentaje de estudios de casos que reportan cada uno de los principios de Ostrom.

Fuente: propia. 
De acuerdo con la identificación y manifestación de los elementos sistematizados sobre los principios propuestos por Ostrom (2011), el relacionamiento y gestión del bosque presenta características que permiten realizar una asociación, según el ámbito de gestión en el que encuentre dicho principio. Los grupos propuestos desde el análisis de los mecanismos encontrados en los casos de estudio nos permiten discernir los siguientes principios: i) los relacionados con las reglas establecidas para la gestión del recurso, así como su aplicación; ii) los mecanismos de seguimiento y monitoreo alrededor del recurso y sus usuarios; iii) los principios que propenden hacia una gobernanza del bosque, entendiendo la gobernanza como mecanismos concertados entre actores.

\section{Reglas y su aplicación}

El principio relacionado con la definición de los usuarios que pueden acceder y usar el recurso es considerado por Ostrom (2011) como uno de los primeros pasos de las organizaciones para la acción colectiva. El $85 \%$ de los casos revisados, cuentan con los límites de los usuarios definidos para el acceso al recurso boscoso, lo que muestra una marcada participación de los actores sobre este principio, sin desconocer las diferentes formas que motivan a los actores a regular este principio. Por lo anterior, se definieron tres criterios (acuerdos implícitos, explícitos y por administración) que recogen las formas de definir los límites de los usuarios. Los acuerdos implícitos hacen referencia a acuerdos que hacen las comunidades locales respecto a las personas que pueden o no acceder al uso del recurso boscoso, sin estar circunscritos o especificados textualmente; estos acuerdos se dan enmarcados en relaciones de confianza entre usuarios ya que al estar en comunidades usualmente con poblaciones pequeñas, las personas suelen conocerse y contar con cierto prestigio dentro de la comunidad (Gautam \& Shivakoti, 2005;
Pacheco, Ibarra, Cronkleton, \& Amaral, 2008; C. Tucker, 1995). También se dan acuerdos de manejo tradicionales en comunidades principalmente indígenas (Ayma-Romayab, 2012; Becker \& Leon, 2000), en donde las decisiones sobre quienes acceden al recurso y las reglamentaciones sobre el uso del mismo, se trasmiten entre generaciones que han estado en el territorio y por lo tanto tienen derechos consuetudinarios sobre el recurso (Monroy-sais, Castillo, García-frapolli, \& Ibarra-manríquez, 2016; Monroy, 2013; Tejeda Cruz \& Márquez Rosano, 2006).

Los acuerdos explícitos se dan a través de documentos como reglamentos internos, planes de manejo, planes de aprovechamiento o identificaciones como carnets, los cuales especifican las características de las personas que pueden usar el recurso y las consecuentes implicaciones. Estos acuerdos explícitos pueden ser considerados y construidos internamente entre los usuarios o las comunidades locales (Pacheco et al., 2008), pero también pueden ser producto de regulaciones de instituciones que administran el recurso y que son territorializados en los contextos particulares (Beltrán Ruiz, 2013; Salcedo-Hernández, 2011). La administración para la regulación del uso del recurso implica instancias participativas a nivel local y/o instituciones de mayor nivel territorial. En algunos casos las instituciones externas tienen un papel regulatorio, el cual implica una capacidad de decisión de sobre el recurso y su uso a nivel local, generando, en algunos casos, conflictos con los usuarios y las organizaciones locales como se citó en el principio de mecanismos para la resolución de conflictos (Beltrán Ruiz, 2013; Salcedo-Hernández, 2011).

Cuando se habla de límites del recurso se establecen dos categorías: la categoría biofísica se relaciona con factores biológicos o geográficos 
sobre los cuales se toman las decisiones para saber la cantidad máxima que se puede extraer del recurso; complementariamente (estas categorías no son excluyentes) la categoría administrativa recoge los casos en donde las decisiones de regular el uso del recurso de acuerdo con sus condiciones y límites se toma desde instituciones que a menudo usan herramientas de manejo. En el $73 \%$ de los casos se encontró una delimitación geográfica, siendo la principal forma de limitar el recurso a usar, hace referencia a las áreas donde la comunidad tiene potestad o en su sentido estricto, las áreas pertenecientes directamente a la comunidad (Duran, Bray, Velazquez, \& Larrazabal, 2011; Gautam \& Shivakoti, 2005; Salcedo-Hernández, 2011; C. Tucker, 1995). Sin embargo, la mayoría de comunidades llevan a cabo un ejercicio de zonificación de bosques (46\%) (Beltrán Ruiz, 2013; Bojórguez-Vargas, Bello-Baltazar, Márquez-Rosano, Cayuelo-Delgado, \& Parra-Vázguez, 2009; Elias, Larson, \& Mendoza, 2009; Salcedo-Hernández, 2011). La administración (42\%) sobre los límites de los recursos se da generalmente a escalas locales donde los consejos, asociaciones, asambleas y demás, son los que participativamente toman las decisiones sobre las cantidades de recurso a aprovechar y formas de aprovecharlo (Ayma-Romayab \& Castro, 2013; Candela, Stoian, Somarriba, \& Villalobos, 2013; C. Tucker, 1995). De la misma forma, ciertos grupos que tienen relaciones particulares con el territorio definen los límites a través de prácticas ancestrales y sistemas de conocimientos propios (Ghate \& Nagendra, 2005). Externamente, instituciones dedicadas a la regulación forestal nacional, emiten normativas y delimitan los bosques de acuerdo a características e intereses estatales. Estas formas de decisión complementan, en ciertos casos, a las formas internas (Beltrán Ruiz, 2013); sin embargo, en otros casos se generan conflictos por oposición de visiones entre actores e instituciones
(Salcedo-Hernández, 2011). Considerando las anteriores formas de administración, cabe resaltar que hay una correspondencia entre estas instituciones con herramientas de manejo (42\%) que permiten la concreción de las normativas y decisiones de gestión. Las herramientas reportadas son planes de manejo, reglamentos, planes de manejo forestal, planes silvícolas, licencias.

De acuerdo con lo documentado en los estudios de caso, los mecanismos para la resolución de conflictos, se realizan a través de mecanismos internos, propios de las comunidades o externos a ellas. Los mecanismos internos predominan dentro de los casos revisados (46\%), puesto que el conocimiento entre usuarios, la confianza y las instancias internas de participación permiten que allí mismo se solucionen los conflictos de menor grado que se presenten (Barton \& Merino, 2004; Barton, Merino, \& Barry, 2007; Beltrán Ruiz, 2013; Bojórguez-Vargas et al., 2009; Candela et al., 2013). Por fuera del orden local, los mecanismos externos son instancias de justicia ordinaria, tribunales de justicia, policía y en algunos casos la iglesia (Ghate \& Nagendra, 2005). Algunas ONG's apoyan las comunidades locales generando espacios de discusión para que se lleguen acuerdos sobre la solución de los conflictos (Ayma-Romayab \& Castro, 2013). Así mismo se evidenció que en algunos casos donde las reglas son impuestas externamente y si conocimiento, llevan al incumplimiento de las mismas y por lo tanto emergen conflictos alrededor (Gautam \& Shivakoti, 2005).

El principio de sanciones graduadas se compone de sanciones internas de las comunidades y otras externas, sin embargo, no se hace referencia a que alguna de estas sea la que se debe aplicar, sino por el contrario, al ser sanciones graduadas debe darse un sistema sancionatorio claro, en articulación entre 
instituciones. Un alto número de casos reportan no tener sanciones graduadas (35\%), lo que responde a la falta de comprensión sobre las normas de gestión de los recursos, teniendo una respuesta subjetiva por cada caso presentado (Gautam \& Shivakoti, 2005; Ghate \& Nagendra, 2005; C. Tucker, 1995). El $46 \%$ de los casos reportaron tener sanciones que se imparten de forma interna por las instituciones locales o por los mismos usuarios. Las sanciones aunque en algunos casos suelen ser graduadas no llegan a un nivel donde intervenga la justicia ordinaria; únicamente participan las instituciones locales con sus sistemas sancionatorios. Estos sistemas buscan que los usuarios que cometan faltas sean conocidos por toda la comunidad, esto con base en el prestigio y con el objetivo de no repetición (Ayma-Romayab \& Castro, 2013; Beltrán Ruiz, 2013; Bojórguez-Vargas et al., 2009; Elias et al., 2009; Márquez, 2011; Pérez, 2010). Las sanciones externas (38\%) son generalmente impuestas por instituciones de mayor nivel territorial que velan por el adecuado uso de los recursos. Se reportan para algunos casos la articulación con las instituciones internas para actuar en el momento que estas lo soliciten, teniendo claridad sobre las sanciones y los niveles de estas (Candela et al., 2013; Elias et al., 2009; Regidor, 2010). Sin embargo también se presentan casos en los que estas instituciones solo actúan directamente sobre los usuarios que comenten faltas (Beltrán Ruiz, 2013; Salcedo-Hernández, 2011).

Las reglas de apropiación se relacionan con las unidades del recurso a adquirir y las variables que se establecen en esta interacción. Las reglas internas (65\%), son creadas y/o heredadas por los mismos usuarios del recurso representados a través de distintas instancias de toma de decisiones locales. Estas decisiones de reglamentación parten de un conocimiento arraigado del bosque, en donde muchas veces no solo se tienen en cuenta el bosque por si mismo, sino el sistema en el que está inmerso; aunque se prioriza el uso de subsistencia frente a las reglamentaciones impuestas en varios de los casos analizados (Bojórguez-Vargas et al., 2009; Elias et al., 2009; Ghate \& Nagendra, 2005; Pacheco, Barry, Cronkleton, \& Larson, 2009), también hay reglas para aprovechamientos comerciales en donde se tienen en cuenta variables propias de las especies por comercializar (Ayma-Romayab \& Castro, 2013; Beltrán Ruiz, 2013; Salcedo-Hernández, 2011; C. Tucker, 1995). Aunque hay casos donde se manifiesta que la reglamentación para la apropiación del recurso responde a reglas externas de las comunidades, su porcentaje es bajo ( $4 \%)$, estas son reglas macro que aunque velan por la protección del recurso, los usuarios manifiestan que no son coherentes con los contextos locales y por lo tanto no las consideran legítimas (Beltrán Ruiz, 2013; Gautam \& Shivakoti, 2005).

A través de las reglas de provisión se busca cuidar el recurso o los beneficios que se obtienen de este, por lo tanto se prevé como una forma de compensación, frente a lo anterior cuando se habla de recuperación por funcionalidad (46\%) hace referencia a acciones que ayudan a recuperar el recurso y su funcionalidad. Por lo tanto la restauración y reforestación específica a los contextos que se analizaron con los casos son la base para trabajar en la provisión del bosque (Elias et al., 2009; Ghate \& Nagendra, 2005; Salcedo-Hernández, 2011). El criterio de provisión natural reglada (38 \%) atañe acciones de uso del recurso que impliquen tasas y compensaciones para permitir el uso del mismo (Bojórguez-Vargas et al., 2009; Elias et al., 2009; Gautam \& Shivakoti, 2005; Pacheco et al., 2009). Por último, las reglas de provisión natural por funcionalidad (46\%) son acciones que contribuyen a mantener sosteniblemente el recurso, que van 
desde el mejoramiento de vías de extracción, tiempos de corta, investigación y la participación permanente de los usuarios del recurso, en el mantenimiento del mismo (Beltrán Ruiz, 2013; Ghate \& Nagendra, 2005; Salcedo-Hernández, 2011).

\section{Procesos de seguimiento y monitoreo}

El monitoreo del recurso se da a partir del seguimiento a las actividades de aprovechamiento o del seguimiento de las condiciones del bosque. Las actividades de aprovechamiento (27\%) son en su mayoría realizadas por los propios usuarios en las instancias organizativas locales (Candela et al., 2013; Duran et al., 2011; Gautam \& Shivakoti, 2005; Ghate \& Nagendra, 2005), sin embargo en algunos casos se responde a normativa impuesta desde instituciones externas que llega a monitorear el recurso (Salcedo-Hernández, 2011). Se monitorea principalmente las especies que se van a extraer y se controlan los focos de incendios para así evitar las pérdidas de recursos. El monitoreo de las condiciones del bosque (35\%) se hace por alguna institución externa a la comunidad, aunque esta no resulta ser la generalidad, pues son los mismos usuarios los encargados de monitorear el recurso (AymaRomayab \& Castro, 2013; Salcedo-Hernández, 2011). Dentro de las comunidades, las instancias organizativas se reúnen para analizar los cambios en el bosque y tomar decisiones sobre los mismos (Bojórguez-Vargas et al., 2009; Bray, 2004; Ghate \& Nagendra, 2005).

El monitoreo de las personas que usan el recurso se presenta en instancias locales y externas. El monitoreo local (54 \%) se realiza a través de las diferentes instancias organizativas creadas en las comunidades locales, siendo los mismos usuarios los que monitorean el recurso, con base en normas establecidas localmente, así como normas externas que se adaptan por las comunidades para ser aplicadas y monitoreadas internamente (Ayma-Romayab \& Castro, 2013; Becker \& Gibson, 1998; Beltrán Ruiz, 2013; Bojórguez-Vargas et al., 2009; Candela et al., 2013; Elias et al., 2009; Gautam \& Shivakoti, 2005; Ghate \& Nagendra, 2005; Márquez, 2011; Pérez, 2010). El criterio de monitoreo externo (12\%), se da por parte de instituciones que administran los recursos a niveles regionales o de país, son ellos quienes definen quienes pueden acceder y usar el recurso y quiénes no; sin embargo, para buscar más efectividad en esta labor, suelen contratar a personas propias de las comunidades para que sean ellas directamente las que monitoreen el recurso (Salcedo-Hernández, 2011).

\section{Interacción para una gobernanza efectiva}

El principio de arreglos de elección colectiva se evidencia en la participación de los usuarios en la toma de decisiones sobre los recursos y se relaciona con la existencia de instituciones locales que velan por este derecho; sin embargo, cuando se observa desde una escala institucional mayor, la participación resulta baja, dejando a un lado el principio básico de la gobernanza, el cual implica una participación y concertación con los usuarios locales en escalas mayores de decisión. Los arreglos definidos por los usuarios (77 \%), se dan en los entornos de las comunidades, donde los usuarios de los recursos suelen reunirse a tomar decisiones sobre la forma en la que se debe usar el recurso (Ayma-Romayab \& Castro, 2013; Cardona, de Jong, Zuidema, \& Boot, 2014; Gautam \& Shivakoti, 2005; Salcedo-Hernández, 2011). Como se indicó en principios anteriores, los grupos de usuarios crean instituciones locales (65\%) que velan por el bienestar de sus comunidades y por el correcto manejo de los recursos; estas instituciones son creadas de acuerdo a las formas y los principios que regulan la relación de los usuarios con sus recursos y la participación de 
los usuarios es permanente y de forma rotativa sobre los cargos. Las instituciones propician los diferentes espacios de participación interna (solo participantes de la institución) o extendida (toda la comunidad) para definir las reglas y las modificaciones sobre las mismas (Barrientos, 2011; Beltrán Ruiz, 2013; Bojórguez-Vargas et al., 2009; Candela et al., 2013; Elias et al., 2009; Ghate \& Nagendra, 2005; Pacheco et al., 2009; Regidor, 2010). Cuando se habla de gobernanza (19\%) en este principio hace referencia a la capacidad de articulación que tienen las instituciones locales con instituciones de mayor nivel, para así lograr unos arreglos colectivos de normas que vayan a favor tanto de los usuarios locales como de los intereses de las instituciones externas. Los casos de estudio que se registraron en este nivel cuentan con la participación de entidades de gobierno en los espacios de toma de decisiones, en donde se llegan a consensos (Elias et al., 2009; Gautam \& Shivakoti, 2005).

El reconocimiento de derechos de organización hace referencia a la importancia que las instancias de decisiones y de manejo de las organizaciones locales, sean reconocidas por instituciones de mayor nivel, teniendo la posibilidad de un mayor reconocimiento en la construcción de acuerdos de gestión y de marcos normativos acordes con las realidades locales. El hecho de desconocer las organizaciones y sus acuerdos genera en muchos casos que las políticas de gestión no sean acordes con la realidades locales, propiciando conflictos entre la normativa y el uso del recurso (Bottazzi \& Dao, 2013; Nayak, 2002). Las instituciones internas (42\%) son aquellas que se encuentran en un mismo nivel de toma de decisiones, por lo tanto, para la mayoría de casos reportados, existen organizaciones paralelas en el territorio que también hacen parte de la gestión de los recursos, estas instituciones se reconocen mutuamente pues llegan a complementarse tanto como figuras de gestión en el territorio (Beltrán Ruiz, 2013; Bojórguez-Vargas et al., 2009; Elias et al., 2009; Pacheco et al., 2009). El reconocimiento desde las instituciones externas $(27 \%)$ se da en organizaciones que se encuentran en un mismo nivel y en menor medida en instituciones de un nivel más alto, de hecho para que haya este reconocimiento en algunos caso se le pide a las organizaciones que modifiquen su reglamento (Becker, 2003; Gautam \& Shivakoti, 2005; Ghate \& Nagendra, 2005; Márquez, 2011; Oberlack \& Schmerbeck, 2015; Salcedo-Hernández, 2011).

En el principio de entidades anidadas se da un anidamiento a nivel local (31\%) permitiendo que haya una articulación para el trabajo en temas comunes, así mismo en este nivel se presenta articulación con el municipio y las instituciones directamente implicadas en las actividades cotidianas de los grupos de usuarios (Ayma-Romayab \& Castro, 2013; Barrientos, 2011; Bojórguez-Vargas et al., 2009; Bray, 2004; Salcedo-Hernández, 2011). Las entidades anidadas a nivel nacional (27\%), se presentan principalmente en la asociación de los usuarios del bosque con entidades gubernamentales que actúan para la regulación del recurso forestal. El papel de las organizaciones externas radica básicamente en la regulación sobre los procesos de aprovechamiento y monitoreo de los productos del bosque (Beltrán Ruiz, 2013; Candela et al., 2013; Elias et al., 2009). En menor medida se acompaña la formulación de normativa a nivel local, lo que genera conflictos en la aplicación y cumplimiento de normas por no contemplar las realidades locales que se encuentran comúnmente en los territorios. Los conflictos en la gobernanza se dan cuando no hay entidades anidadas (42\%), situación que se presenta en muchos casos pues la posibilidad de la inclusión de visiones en la creación de regímenes de gestión se ve limitada. Por un lado, están 
los actores externos con marcos regulatorios de difícil aplicación en lo local y por el otro los grupos de usuarios con claras necesidades que solucionar de acuerdo a sus posibilidades y a los patrones de manejo históricamente adquiridos (Pacheco et al., 2009). La anidación de entidades debe contemplar la participación conjunta en las estrategias de manejo que se incorporen en lo local, pero así mismo nutran de elementos para la creación de marcos regulatorios generales.

\section{CONCLUSIONES}

Los principios elegidos conceptualmente como eje de una gestión efectiva según Ostrom, se agrupan estratégicamente en tres niveles: i) Reglas y su aplicación, ii) Procesos de seguimiento y monitoreo e iii) Interacción para una gobernanza efectiva. La mayoría de parámetros se concentran en definir reglas, emergiendo el seguimiento como un mecanismo de verificación necesario más no incidente en la gestión efectiva del bosque como RUC. La interacción entre actores locales en contacto con el RUC, resulta ser la más abordada en todos los estudios, lo que ratifica las conclusiones de Ostrom, puesto que la efectividad del manejo sostenible de los bosques pasa preponderantemente por la autogestión.

Las reglas son el principio de interacción que más observa heterogeneidad, razón por la cual es la incluye el volumen más alto de parámetros (5), entre los cuales ninguno resulta ser el más observado en las sistematizaciones, sea como factor de éxito en su presencia, o fracaso por estar ausente; no obstante se observa que las reglas son un aspecto definitivo del principio de gestión efectiva, además de ser las que empoderan las estrategias de seguimiento, que con sus resultados retroalimentan las reglas $y$, primordialmente, sustentan una gobernanza efectiva aprobando o desaprobando acciones de relacionamiento.
Es evidente que una gestión efectiva del bosque como RUC, transita por condiciones hechas a la medida de cada contexto, pero responden a los ocho principios establecidos por Ostrom, no obstante, cambian de protagonismo según sean las condiciones refugiándose más en uno que en otro.

\section{LITERATURA CITADA}

Agrawal, A. 2002. Common Resources and Institutional Sustainability. In The Drama of the Commons, National Research Council, Committee on the Human Dimensions of Global Change, ed. Elinor Ostrom, Thomas Dietz, Nives Dolšak, Paul Stern, Susan Stonich, and Elke Weber, 41-85. Washington, DC: National Academy Press

Agrawal, A. (2007). Forests, Governance, and Sustainability: Common Property Theory and its Contributions. International Journal of the Commons, 1(1), 111-136.

Ayma-Romayab, A. I. (2012). Instituciones y autogobernanza del bosque comun de una comunidad Aymara en los andes bolivianos. Universidad de Chile.

Ayma-Romayab, A. I., \& Castro, V. (2013). Causas sociales y ecológicas subyacentes para un mejor manejo comunitario del bosque de neblina en una comunidad aymara de los Andes de Bolivia. Bolivia.

Barton, D., \& Merino, L. (2004). La experiencia de las comunidades forestales en México. Veinticinco años de silvicultura y construcción de empresas forestales comunitarias. Mexíco DF: Instituto Nacional de Ecología (INE-SEMARNAT).

Barton, D., Merino, L., \& Barry, D. (Eds.). (2007). Los bosques comunitarios de México, Manejo sustentable de paisajes forestales (Primera ed). Mexíco DF: Instituto Nacional de Ecología (INE-Semarnat).

Becker, D. (2003). Grassroots to grassroots: Why forest preservation was rapid at Loma Alta, Ecuador. World Development, 31(1), 163-176.

Becker, D., \& Gibson, C. (1998). The lack of Institutional supply: why a strong local community in western Ecuador fails to protect its forest. In C. Gibson, M. McKean, \& E. Ostrom (Eds.), Forest resources and institutions. Forests, Trees and People Programme Working Paper 3 (p. 23). Rome, Italy: FAO. 
Becker, D., \& Leon, R. (2000). Indigenous Forest Management in the Bolivian Amazon: Lessons from the Yuracare People. In C. C. Gibson, M. McKean, \& E. Ostrom (Eds.), People and Forest: Communities, institutions and governance (pp. 163-191). Indiana: MIT Press.

Beltrán Ruiz, A. (2013). Recursos del bosque comunitario irecursos de todos?: Análisis del uso y manejo de los recursos de uso común desde la perspectiva de género en un consejo comunitario del Chocó Biogeográfico, Consejo Comunitario Mayor del Alto San Juan (ASOCASAN). Pontificia Universidad Javeriana.

Bojórguez-Vargas, A. R., Bello-Baltazar, E., Márquez-Rosano, C., Cayuelo-Delgado, L., \& ParraVázguez, M. (2009). Forestería comunitaria y desarrollo de instituciones locales: el caso de la Comunidad Agraria Teopisca. Economía Sociedad Y Territorio, 9(30), 349-395.

Bollier, D. (2014). Pensar desde los comunes- Una breve introducción. Canada: New Society Publishers, Gabriola Island.

Bottazzi, P., \& Dao, H. (2013). On the road through the Bolivian Amazon: A multi-level land governance analysis of deforestation. Land Use Policy, 30(1), 137-146.

Candela, S., Stoian, D., Somarriba, E., \& Villalobos, M. (2013). Formas y efectos de la gobernanza forestal en los territorios indígenas bribri y cabécar de Alta Talamanca, Costa Rica. Agroforestería En Las Américas, 49, 85-92.

Cardenas, J. C. (2009). Dilemas de lo colectivo: instituciones, pobreza y cooperación en el manejo local de los recursos de uso común. Bogotá D.C., Colombia.: Universidad de los Andes, Facultad de Economía, CEDE, Ediciones Uniandes.

Cardona, W. C., de Jong, W., Zuidema, P. A., \& Boot, R. (2014). Diverse local regulatory responses to a new forestry regime in forest communities in the Bolivian Amazon. Land Use Policy, 39, 224-232.

Cox, M., Arnold, G., \& Villamayor, S. (2010). A Review of Design Principles for Community-based Natural Resource Management. Ecology and Society, 15(4), 19.

Delgado Riaño, A. (2017). La conciencia de lo común frente a la falsa dicotomía estado-mercado Análisis de la perspectiva de Elinor Ostrom (1933-2012). Universidad Complutense de Madridnto de Historia e Instituciones Económicas.

Duran, E., Bray, D. B., Velazquez, A., \& Larrazabal,
A. (2011). Multi-Scale Forest Governance, Deforestation, and Violence in Two Regions of Guerrero, Mexico. World Development, 39(4), 611-619.

Elias, S., Larson, A., \& Mendoza, J. (2009). Tenencia De La Tierra, Bosques Y Medios De Vida En El Altiplano Occidental De Guatemala. Editorial de Ciencias Sociales. Universidad de San Carlos de Guatemala.

Gautam, A. P., \& Shivakoti, G. P. (2005). Conditions for Successful Local Collective Action in Forestry: Some Evidence From the Hills of Nepal. Society \& Natural Resources, 18(April), 153-171.

Ghate, R., \& Nagendra, H. (2005). Role of Monitoring in Institutional Performance: Forest Management in Maharashtra, India. Conservation and Society, 3(2), 509.

Gibson, C. C., Williams, J. T., \& Ostrom, E. (2005). Local enforcement and better forests. World Development, 33(2), 273-284.

Glaser, B. \& Strauss, A. (1967). The discovery of grounded theory. Chicago: Aldine Press.

Gómez, F., \& Guerrero, H. (2014). El análisis institucional en el campo de la gestión de los recursos naturales. Económia Y Sociedad, 30, 67-86.

Hardin, G. (1968). The tragedy of the commons. Science, 162, 1243-1248. Heckathorn

Márquez, L. (2011). The Effect Of Institutions On Guatemalan Forests: Conceptual, methodological and practical implications. Indiana University.

Meinzen-Dick, R. S., \& Di Gregorio, M. (2004). Acción colectiva y derechos de propiedad para el desarrollo sostenible. CAPRi, PROGRAMA PARA TODO EL SISTEMA DEL CGIAR SOBRE ACCIÓN COLECTIVA Y DERECHOS DE PROPIEDAD.

Monroy-sais, S., Castillo, A., García-frapolli, E., \& Ibarra-manríquez, G. (2016). Ecological variability and rule-making processes for forest management institutions : a social-ecological case study in the Jalisco coast, Mexico. International Journal of the Commons, 10(2), 1144-1171.

Monroy, S. (2013). Historia, uso y manejo de los bosques en un ejido de la región Chamela-Cuixmala, Jalisco. Universidad Nacional Autónoma De México.

Nayak, P. K. (2002). Community-Based Forest Management In India: The Issue Of Tenurial Significance. In Paper for the 9th Biennial Conference of the IASCP (pp. 1-35). Zimbabwe: Foundation for Ecological Security PO. 
Oberlack, C., \& Schmerbeck, J. (2015). Institutions for sustainable forest governance: Robustness, equity, and cross-level interactions in Mawlyngbna, Meghalaya, India. International Journal of the Commons, 9(2), 670-697.

Olivos, R. A. (2014). Bienes comunes y democracia, Hermenéutica crítica de las instituciones del procomún. Universidad de Valencia.

Olson, M. (1965). The logic of collective action: Public goods and the theory of groups. Cambridge, MA: Harvard University Press

Ostrom, Elinor. (1990). Governing the Commons: The Evolution of Institutions for Collective Action. New York: Cambridge University Press.

Ostrom, E. (2009). A general framework for analyzing sustainability of social-ecological systems. Science (New York, N.Y.), 325(5939), 419-422.

Ostrom, E. (2009a). Design principles of robust property-rights institutions: what have we learned? Property Rights and Land Policies, 8(19), 1-28.

Ostrom, E. (2009b). Las reglas que no se hacen cumplir son mera palabreria. Revista de Economia Institucional, 11(21), 15-24.

Ostrom, E. (2009c). Más allá de los mercados y los Estados: gobernanza policéntrica de sistemas económicos complejos (pp. 15-70).

Ostrom, E. (2011). El gobierno de los bienes comunes. La evolución de las instituciones de acción colectiva. FCE. UNAM. Mexico. 402p.

Ostrom, E., \& Nagendra, H. (2006). Insights on linking forests, trees, and people from the air, on the ground, and in the laboratory. Proceedings of the National Academy of Sciences of the United States of America, 103(51), 19224-31.

Pacheco, P., Barry, D., Cronkleton, P., \& Larson, A. M. (2009). El papel de las instituciones informales en el uso de los recursos forestales en América Latina. Indonesia: CIFOR.

Pacheco, P., Ibarra, E., Cronkleton, P., \& Amaral, P. (2008). Políticas públicas que afectan el manejo forestal comunitario. In Manejo Forestal Comunitario en América Latina: Experiencias, lecciones aprendidas y retos para el futuro (pp. 201-230). Belém, Brasil: CIFOR - CATIE.

Pérez, M. R. (2010). Las instituciones comunitarias en el manejo de los recursos forestales en la comunidad de San Andrés Yatuni, Oaxaca (México). Universidad Politécnica De Madrid.

Poteete, A., Janssen, M., \& Ostrom, E. (2012). Trabajar Juntos: Acción colectiva, bienes comunes y múltiples métodos en la práctica. Mexico: UNAM, CEIICH, CRIM, FCPS, FE, IIEC, IIS, PUMA; IASC, CIDE, Colsan, CONABIO, CCMSS, FCE, UAM.

Salcedo-Hernández, D. (2011). Análisis de la acción colectiva en el uso y manejo del mangle en la zona de uso sostenible del sector estuarino de la bahía de Cispatá en el departamento de Córdoba. Pontificia Universidad Javeriana.

Schlager, E., \& Ostrom, E. (1992). Property-Rights Regimes and Natural Resources: A Conceptual Analysis. Land Economics, 68(3), 249-262.

Tejeda Cruz, C., \& Márquez Rosano, C. (2006). Apropiación territorial y aprovechamiento de recursos forestales en la comunidad Frontera Corozal, Selva Lacandona, Chiapas, México. Revista Geografía Agrícola, 37(August 2004), 79-95.

Tucker, C. (1995). Evaluating a Common Property Institution: Design Principles and Forest Management in a Honduran Community. CIPEC. Indiana.

Tucker, C. M. (2010). Learning on Governance in Forest Ecosystems : Lessons from Recent Research. International Journal of the Commons, $4(2), 687-706$.
Conflicto de Intereses Los autores declaran no tener ningún conflicto de intereses 
